\title{
Simultaneous implementation of low dose and high sensitivity capabilities in differential phase contrast and dark-field imaging with laboratory $x$-ray sources
}

\author{
A. Olivo*, C. K. Hagen, T. P. Millard, F. Vittoria, P. C. Diemoz, M. Endrizzi \\ Department of Medical Physics and Bioengineering, University College London, United Kingdom
}

\begin{abstract}
We present a development of the laboratory-based implementation of edge-illumination (EI) x-ray phase contrast imaging (XPCI) that simultaneously enables low-dose and high sensitivity. Lab-based EI-XPCI simplifies the set-up with respect to other methods, as it only requires two optical elements, the large pitch of which relaxes the alignment requirements. Albeit in the past it was erroneously assumed that this would reduce the sensitivity, we demonstrate quantitatively that this is not the case.

We discuss a system where the pre-sample mask open fraction is smaller than $50 \%$, and a large fraction of the created beamlets hits the apertures in the detector mask. This ensures that the majority of photons traversing the sample are detected i.e. used for image formation, optimizing dose delivery. We show that the sensitivity depends on the dimension of the part of each beamlet hitting the detector apertures, optimized in the system design. We also show that the aperture pitch does not influence the sensitivity. Compared to previous implementations, we only reduced the beamlet fraction hitting the absorbing septa on the detector mask, not the one falling inside the apertures: the same number of $\mathrm{x}$-rays per second is thus detected, i.e. the dose is reduced, but not at the expense of exposure time.

We also present an extension of our phase-retrieval algorithm enabling the extraction of ultra-small-angle scattering by means of only one additional frame, with all three frames acquired within dose limits imposed by e.g. clinical mammography, and easy adaptation to lab-based phase-contrast x-ray microscopy implementations.
\end{abstract}

Keywords: edge illumination, coded apertures, phase contrast, $x$-ray imaging, phase sensitivity, dark field imaging.

\section{INTRODUCTION}

It is well known that being able to gain access to phase, and possibly dark field (i.e. ultra-small angle scattering) information alongside absorption could radically transform $x$-ray imaging, both in medical applications ${ }^{1}$ and beyond. ${ }^{2}$ While nowadays most x-ray phase contrast imaging (XPCI) methods can be considered well-established synchrotron tools, research is still directed towards the development of effective, robust and efficient implementations with laboratory sources, as these would widely expand access to XPCI methods to researchers worldwide, and enable transition of the technology into clinical and other environments.

Effectively, experiments with laboratory sources have been carried out since the early days of the method; ${ }^{3,4}$ however, the typical downside is that of excessive exposure times, up to hours. ${ }^{4}$ This is due to the restrictive coherence requirements XPCI methods impose on the radiation sources, especially in terms of spatial coherence: for example, phase enhancements brought by free-space propagation approaches, ${ }^{4,5}$ vanish rapidly as the source size is made larger. ${ }^{6}$ Albeit exceptions exist (e.g. crystal-based methods can tolerate more spatial than temporal coherence ${ }^{7}$ ), ultimately similar problems are encountered - e.g., in the quoted example, the crystal automatically selects a very narrow component of the available spectral bandwidth, again leading to excessive exposure times.

A promising attempt at solving the problem was recently developed when the Talbot-Lau modification ${ }^{8}$ of the $\mathrm{x}$-ray Talbot interferometer ${ }^{9}$ was proposed by Pfeiffer et al. This method was rapidly and deservedly taken on by a large number of groups worldwide, as it enables for the first time the use of extended sources. It should be noted, however, that the method is still intrinsically coherent: an incoherent source is made sufficiently coherent by the introduction of the source grating, which is the basis of the transition from the Talbot to the Talbot-Lau configuration.

*a.olivo@ucl.ac.uk; phone +44 20 7679-2444; fax +44 20 7679-0255 
Although the method goes a long way in solving the limitations of previous approaches, it still suffers from some shortcomings, which we find are due to its intrinsically coherent nature. A partial list includes the necessity of using a source grating, which leads to an inefficient use of the radiation emitted from the source; the demanding alignment requirements ${ }^{10}$, due to the small grating pitch and the need for phase-stepping approaches; the delivery of doses which can be several orders of magnitude above clinical practice, ${ }^{11}$ due to the radiation absorbed in the post-sample gratings. With regards to this, it should be noted that, while most authors focus on the absorption nature of the last, "analyzer" grating, which in principle would only absorb $50 \%$ of the radiation, a more significant role is played by the $\sim 300-500 \mu \mathrm{m}$ thick silicon substrates both the phase and the analyzer grating are built on: at e.g. mammographic energies, these absorb a considerable fraction of the radiation after it has traversed the sample. While in principle it would be possible to thin them down, this is in conflict with the requirements to build gratings with larger areas, as they could become excessively brittle.

Our aim is to remove all these shortcomings through the development of a truly incoherent method with the same phase sensitivity. As described below, this can be achieved by using x-ray masks rather than gratings, with a much lower aspect ratio that enables, among other things, the use of graphite rather than silicon substrates, thus achieving a substantially increased x-ray transparency.

\section{MATERIALS AND METHODS}

We propose a new implementation of the edge-illumination method, first developed at the Elettra synchrotron in Italy in the late ' $90 \mathrm{~s} .{ }^{12}$ The method has been adapted for use with incoherent sources (so far, up to $100 \mu \mathrm{m}$ in focal spot size, and with a full polychromatic spectrum with no filtration), without requiring a source grating or collimator, by means of a pair of apertured x-ray masks placed either side of the imaged sample. ${ }^{13}$ Early set-ups ${ }^{14}$ enabled the demonstration of reduced exposure times and robustness against environmental vibrations; later developments ${ }^{15}$ provided hints of lowdose and high sensitivity potential: for example, it can be observed that the signal arising at the cartilage/fluid interface in ref $^{15}$ has the same relative intensity as that reported by Stutman et al ${ }^{16}$ using grating interferometry on the $3^{\text {rd }}$ Talbot order. We now expand on these developments through a new system design that allows the same sensitivity as grating interferometers at a lower dose, and we demonstrate this by measuring experimentally both dose and sensitivity. A schematic representation of the set up modifications is shown in figure 1 .

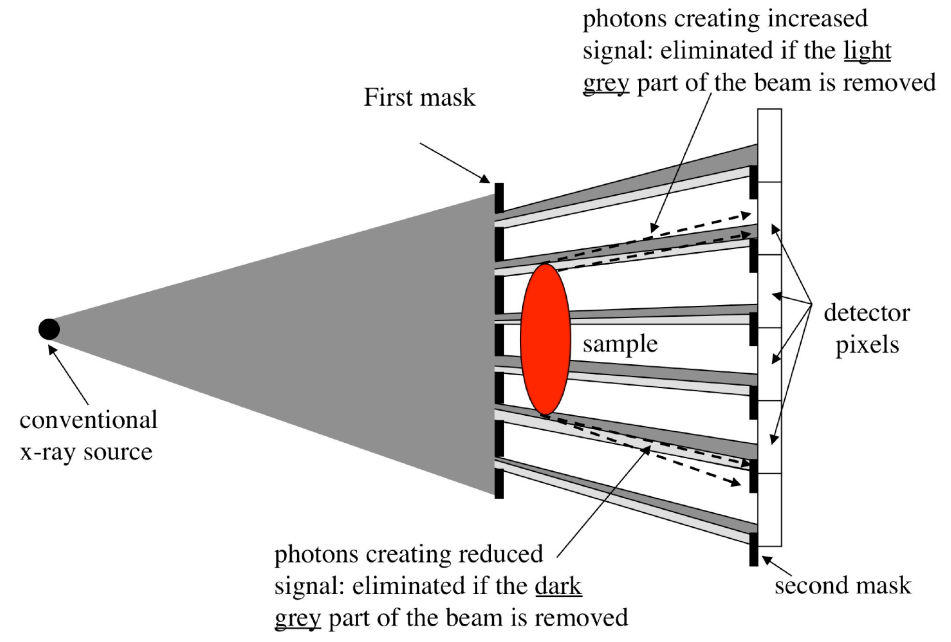

Figure 1. Conceptual modification of the lab-based EI setup. For ease of explanation, the beamlets have been "color-coded" after shaping by the pre-sample mask. The dark grey parts of the beamlets reach the sensitive detector regions, while the light grey ones do not. The dark grey parts can be deviated outside the pixel active surface (=decreased counts): they are thus responsible for the negative peaks in the differential XPCI signals. The light grey parts can be deviated inside the sensitive regions: they give rise to the positive peaks. The "low dose" version of the set-up is obtained by reducing the presample aperture size in the direction coming from below in the drawing i.e. reducing the light grey parts of the beamlets. This can affect the positive peaks, while leaving the negative ones (and the detected background counts) unchanged. Note that, by halving the size of pre-sample apertures and changing the alignment so that either the dark grey or the light grey parts are eliminated, we could effectively create "positive peak only" or "negative peak only" XPCI profiles. 
The new set-up was used to image a number of different samples among which, notably, in vitro breast tissue specimens containing tumors of various types ${ }^{17}, 18$. The dose was measured by replacing the samples with a calibrated Keithley $35050 \mathrm{~A}$ ion chamber (i.e. by placing the chamber immediately downstream of the pre-sample mask). Dose measurements were repeated using termoluminescent dosimeters (TLD-100H) and consistent values were found, to within $10 \%$ accuracy. For comparison purposes, images of the same samples were acquired with a clinical full-field digital mammography system (GE Senographe Essential Version ADS 54.11).

The phase sensitivity was measured through an adaptation to EI of the phase sensitivity definition given by Modregger et $a l .{ }^{19}$ The resulting EI-based sensitivity assessment model had been used in the recent past with coherent synchrotron sources, demonstrating unprecedented angular sensitivity of $\sim 2 \operatorname{nrad}^{20}$. This model has now been further developed, to incorporate the effects of the extended source size and beam polychromaticity ${ }^{21}$.

Moreover, we further developed our algorithm enabling quantitative retrieval of phase and absorption through the acquisition of two frames, ${ }^{22}$ by creating a new version that requires the acquisition of just one additional frame to produce the dark field (ultra-small angle scatter) image. ${ }^{18}$ It is based on measuring the illumination curve (obtained by scanning the pre-sample mask, while the rest of the set-up is kept stationary). As recently demonstrated, in EI this plays the same role as the rocking curve in analyzer based imaging: ${ }^{23}$ however, the absence of the crystal means that the same effect can be obtained with a divergent and polychromatic beam. This curve is measured without the sample; three points on the same curve (i.e. three frames) are then acquired with the sample in place. Various approaches are then available to extract attenuation, scatter and refraction from these three frames. ${ }^{18}$ For example, if the convolution between the illumination curve and the sample scattering function can be approximated by a Gaussian of amplitude $A$, the intensity $I$ at each mask displacement (i.e. illumination curve position) $x_{i}$ can be written as:

$$
I=t \frac{A}{\sqrt{2 \pi \sigma^{2}}} \exp \left[-\frac{\left(x_{i}-\Delta x_{R}\right)^{2}}{2 \sigma^{2}}\right]
$$

where $t$ is the sample transmission, $\Delta x_{R}$ the lateral beamlet displacement (refraction), $\sigma$ the standard deviation expressing the broadening of the illumination curve after traversing the sample. By taking three such images at different values of $x_{i}$, one obtains a system of equations that can be solved for $\mathrm{t}, \Delta x_{R}$ and $\sigma$. If the Gaussian approximation does not hold, the system can still be solved numerically, by using the measured illumination curve as a reference.

Finally, we have implemented a large magnification version of the method by designing a highly minified (10x) presample mask, and placing it at $\sim 10 \mathrm{~cm}$ from the source and $1 \mathrm{~m}$ from the detector. While so far the Rigaku M007 source (which has a focal spot of $\sim 70 \mu \mathrm{m}$ ) was used, for the high magnification measurements we replaced it with a transmission target $\mathrm{W}$ source, with a smaller $(\sim 4 \mu \mathrm{m})$ focal spot. With the Rigaku, the projected source size (i.e. focal spot size times sample-to-detector divided by source-to-sample distance) of $\sim 700 \mu \mathrm{m}$ would have mixed the beamlets together, preventing the realization of the EI condition. While this had an impact on the available flux, the fact that we have also significantly reduced the overall system length (from $2 \mathrm{~m}$ to $1.1 \mathrm{~m}$ ) meant that exposure times went up from seconds to minutes, and not from seconds to hours as one might have feared. Acquisition times of several hours are common in x-ray microscopy, albeit typically in CT set-ups: ${ }^{24}$ in future micro-CT developments, our method should therefore be able to provide comparable if not improved exposure time performance. A different detector was also used in this case, i.e. the Hamamatsu C9732DK CMOS-based flat panel rather then the direct conversion a-Se ANRAD LMAM flat panel.

\section{RESULTS}

In all imaged samples, features invisible by conventional methods methods were detected. For example, we compared calcification detection obtained with our method to that of a standard clinical unit (GE Senographe Essential Version ADS 54.11), by taking images of the same samples at comparable doses, which enabled us to demonstrate that a significantly increased number of calcifications is detected by our XPCI system. ${ }^{17}$ For calcifications visible also with the conventional system, contrast enhancements of up to $10 \mathrm{x}$ were regularly observed, despite the use of detectors with 
roughly the same pixel size (100 $\mu \mathrm{m}$ for the Senographe vs. $85 \mu \mathrm{m}$ for the ANRAD detector used in our study). Entrance doses ranged between 1 and $5 \mathrm{mGy}$, well below the $12 \mathrm{mGy}$ limit imposed for example by EU guidelines. ${ }^{25}$ Although in some cases this was due to the imaged samples being thinner than in the real clinical case, extrapolation to clinically significant breast thicknesses still led to mean glandular dose values fully compatible with clinical requirements. ${ }^{17}$

As far as angular (i.e. differential phase) sensitivity is concerned, we measured this for the lab-based system used to obtain the breast images discussed above, by means of Diemoz et al.'s adaptation ${ }^{20,21}$ of the method developed by Modregger et al. ${ }^{19}$ By using a $7 \mathrm{~s}$ x-ray exposure for each of the two images used in the phase retrieval, this sensitivity was estimated to be of $\sim 270 \mathrm{nrad}$, comparable to if not higher than, for example, that reported by Thüring et al. for grating interferometry, ${ }^{26}$ albeit admittedly for a system with a shorter overall (source-to-detector) length. To the best of our knowledge, the only higher sensitivity value for a lab-based system was reported by Revol et al, who quote a sensitivity of approximately 110 mrad. ${ }^{27}$ It should be noted, however, that in that case 12 frames were acquired, with roughly the same exposure time per frame $(6.7 \mathrm{~s})$ as used by us. Since we are effectively dealing with fluctuations of a background, it is reasonable to expect that, at least to first approximation, this will vary with the square root of the exposure time, as a sheer consequence of Poisson statistics. Interestingly, if one divides our result by the square root of the ratio between ours and Revol et al's exposure time (i.e. $\sqrt{6}$, square root of the ratio between number of frames, as the exposure time per frame is roughly the same), one obtains practically a perfect match $(270 / \sqrt{6} \cong 110)$, which suggests same phase sensitivity. One example of sensitivity for our system is provided in figure 2 .
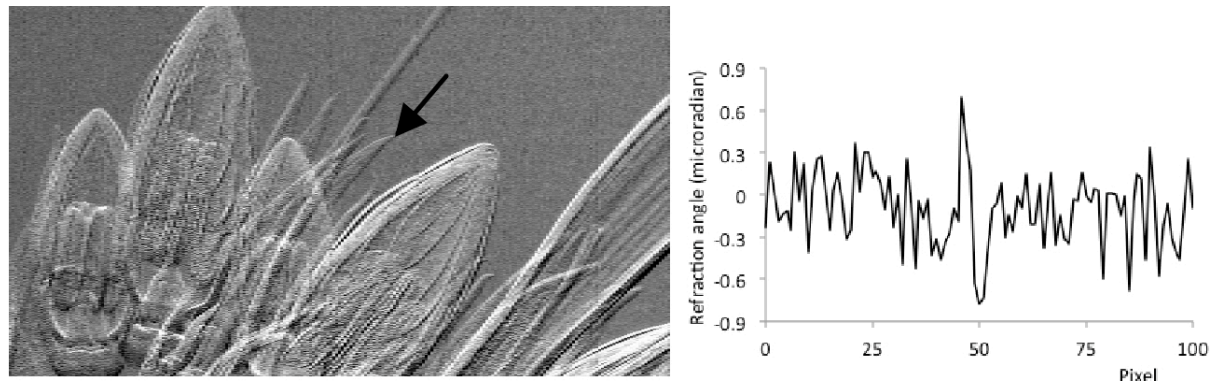

Figure 2. Example of the method's sensitivity. The profile is extracted from the tip of the faint detail of the flower image on the left (see arrow): the angle ( $\mu \mathrm{rad})$ is plotted vs the position in pixels. The detail produces positive/negative peaks both about 700 nrad, clearly distinguishable from the background, which fluctuates by about 2-300 nrads as stated above.

Figure 3 shows an example of application of the three-image retrieval algorithm. This was applied to various samples (including in vitro breast tissue and others ${ }^{18}$ ), and also in this case the full three-image sequence was acquired while delivering total doses compatible with clinical practice. The quantitativeness of the refractive index retrieval was benchmarked both against our previous method, which had already been experimentally validated, ${ }^{22}$ as well as by imaging samples of known composition and comparing the retrieved values against tabulated ones.
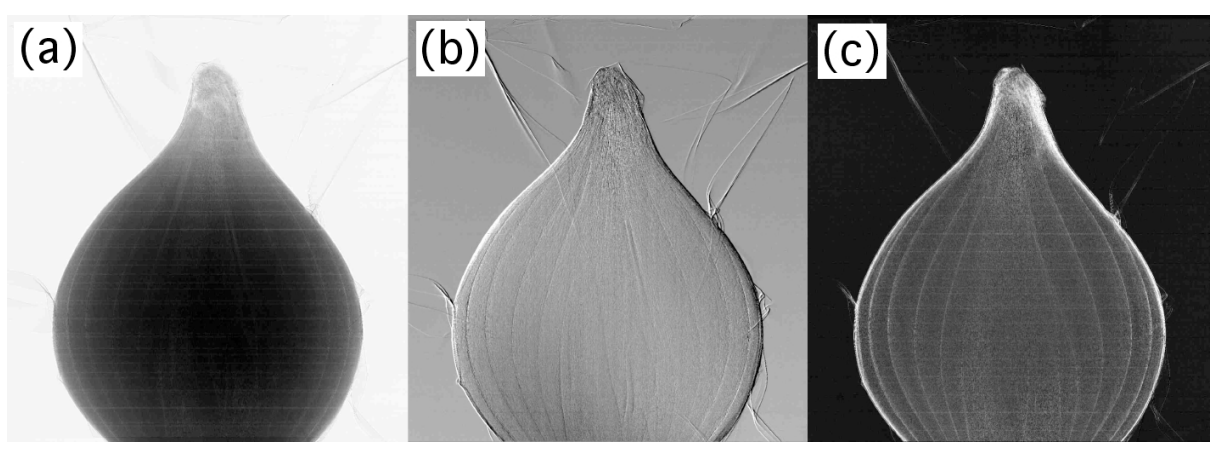

Figure 3. Example of application of the three-image retrieval algorithm to three frames of a sample (in this case a small onion) acquired with different misalignment values between pre-sample and detector masks. (a) attenuation, (b) differential phase contrast and (c) ultra-small angle scattering (dark field) image. 
Finally, figure 4 shows an example image obtained with the microscopic set-up. This was obtained by downscaling the pre-sample mask ten times and using 10x magnification. Albeit the apertures in the pre-sample mask were of $3 \mu \mathrm{m}$, we have experimental evidence that, by "dithering" the sample (i.e. acquiring multiple frames at sub-pixel sample displacements and recombining them to obtain an image with increased spatial resolution ${ }^{13,14}$ ) in steps smaller than the aperture itself, a resolution better than $3 \mu \mathrm{m}$ is achieved. Additional measurements and analysis are currently underway, but this resolution seems to be in the region 1-1.5 $\mu \mathrm{m}$. Phase oscillations inside the $3 \mu \mathrm{m}$ aperture seem to be at the origin of this unexpected resolution enhancement, and a paper on this phenomenon has recently been submitted. ${ }^{28}$

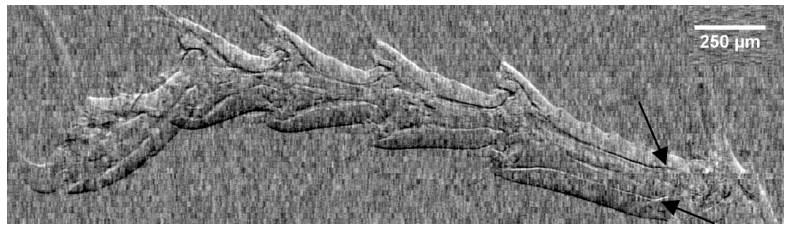

Figure 4. Microscopic image of the leg of a ground beetle. Although the determination of the ultimate system resolution is still to some extent work in progress, arrows point at examples of details with dimensions of the order of $1 \mu \mathrm{m}$. Notably, the tube voltage was of $80 \mathrm{kVp}$, which effectively makes this sample a very weak phase object.

\section{CONCLUSIONS}

We achieved significant new developments in the area of XPCI with the lab-based EI approach. Notably, we have quantitatively measured the phase sensitivity, showing it is at least comparable to that provided by grating interferometry. We showed how the flexibility of the method can be exploited to produce images in which dose is kept to a minimum and is compatible with clinical requirements, without affecting the sensitivity. We have extended our quantitative approach to incorporate dark field imaging, demonstrating that the full retrieval of attenuation, differential phase and ultra-small angle scattering can be carried out with only three frames all of which can be acquired within clinical dose constraints. Finally, we have showed easy adaptation to microscopic set-ups. We believe this shows the great potential of EI XPCI, both as a powerful research tool and as a strong contender for translation into "real world" applications, in medicine and beyond.

\section{ACKNOWLEDGMENTS}

This work is supported by EPSRC (Grant EP/I021884/1). Additional support to P.C. Diemoz and M. Endrizzi is provided through Marie-Curie "Career Integration" grants, awarded within the $7^{\text {th }}$ Framework Programme of the European Union, Grant numbers PCIG12-GA-2012-333990 and PCIG12-GA-2012-334056, respectively.

\section{REFERENCES}

[1] Bravin, A., Coan, P. and Suortti, P., "X-ray phase-contrast imaging: from pre-clinical applications towards clinics," Phys. Med. Biol. 58(1), R1-R35 (2013).

[2] Mayo, S. C., Stevenson, A. W. and Wilkins, S. W., "In-line phase-contrast X-ray imaging and tomography for material science," Materials 5(5), 937-65 (2012).

[3] Davis, T. J., Gao, D., Gureyev, T. E., Stevenson, A. W. and Wilkins, S. W., "Phase-contrast imaging of weakly absorbing materials using hard x-rays," Nature 373(6515), 595-8 (1995).

[4] Wilkins, S. W., Gureyev, T. E., Gao, D., Pogany, A. and Stevenson, A.W., "Phase-contrast imaging using polychromatic hard x-rays." Nature 384(6607), 335-8 (1996).

[5] Snigirev, A., Snigireva, I., Kohn, V., Kuznetsov S. and Schelokov, I., "On the possibilities of x-ray phase contrast microimaging by coherent high-energy synchrotron radiation,” Rev. Sci. Instrum. 66(2), 5486-92 (1995). 
[6] Olivo, A. and Speller R., "Experimental validation of a simple model capable of predicting the phase contrast imaging capabilities of any x-ray imaging system," Phys. Med. Biol. 51(12), 3015-30 (2006).

[7] Vine, D. J., Paganin, D. M., Pavlov, K. M., Kräusslich, J., Wehrhan, O., Uschmann I. and Förster, E., "Analyzer-based phase contrast imaging and phase retrieval using a rotating anode x-ray source," Appl. Phys. Lett. 91(25), 254110 (2007).

[8] Pfeiffer, F., Weitkamp, T., Bunk, O. and David, C., "Phase retrieval and differential phase-contrast imaging with low-brilliance x-ray sources," Nat. Phys. 2(4), 258-61 (2006).

[9] David, C., Nöhammer, B., Solak, H. H. and Ziegler, E., "Differential x-ray phase contrast imaging using a shearing interferometer," Appl. Phys. Lett. 81(17), 3287-9 (2002).

[10]Zambelli, J., Bevins, N., Qui, Z. and Chen, G.-H., "Radiation dose efficiency comparison between differential phase contrast CT and conventional absorption CT," Med. Phys. 37(6), 2473-9 (2010).

[11] Grandl, S., Willner, M., Herzen, J., Mayr, D., Auweter, S. D., Hipp, A., Pfeiffer, F., Reiser, M. and Hellerhoff, K., "Evaluation of phase-contrast CT of breast tissue at conventional x-ray sources - presentation of selected findings," Z. Med. Phys 23(3), 212-21 (2013).

[12] Olivo, A., Arfelli, F., Cantatore, G., Longo, R., Menk, R. H., Pani, S., Prest, M., Poropat, P., Rigon, L., Tromba, G., Vallazza, E. and Castelli, E., "An innovative digital imaging set-up allowing a low-dose approach to phase contrast applications in the medical field," Med. Phys. 28(8), 1610-19 (2001).

[13] Olivo, A. and Speller, R., "Modelling of a novel x-ray phase contrast imaging technique based on coded apertures," Phys. Med. Biol. 52(22), 6555-73 (2007).

[14] Olivo, A., Ignatyev, K., Munro, P. R. T. and Speller, R. D., "Noninterferometric phase-contrast images obtained with incoherent X-ray sources," Appl. Opt. 50(12), 1765-9 (2011).

[15] Marenzana, M., Hagen, C. K., Das Neves Borges, P., Endrizzi, M., Szafraniec, M. B., Ignatyev, K. and Olivo, A., "Visualization of small lesions in rat cartilage by means of laboratory-based x-ray phase contrast imaging," Phys. Med. Biol. 57(24), 8173-84 (2012).

[16] Stutman, D., Beck, T. J., Carrino, J. A. and Bingham, C. O., "Talbot phase-contrast X-ray imaging for the small joints of the hand," Phys. Med. Biol. 56(17), 5697-720 (2011).

[17] Olivo, A., Gkoumas, S., Endrizzi, M., Hagen, C. K., Szafraniec, M. B., Diemoz, P. C., Munro, P. R. T., Ignatyev, K., Johnson, B., Horrocks, J. A., Vinnicombe, S. J., Jones, J. L. and Speller R. D., "Low-dose phase contrast mammography with conventional x-ray sources," Med. Phys. 40(9), 090701 (2013).

[18] Endrizzi, M., Diemoz, P. C., Millard, T. P., Jones, J. L., Speller, R. D., Robinson, I. K. and Olivo A., "Hard xray dark-field imaging with incoherent sample illumination," Appl. Phys. Lett., in press.

[19] Modregger, P., Pinzer, B. R., Thuring, T., Rutishauer, S., David, C. and Stampanoni, M., "Sensitivity of x-ray grating interferometry," Opt. Exp. 19(19), 18324-8 (2011).

[20] Diemoz, P. C., Endrizzi, M., Zapata, C. E., Pesic, Z. D., Rau, C., Bravin, A., Robinson, I. K. and Olivo, A., "Xray phase-contrast imaging with nanoradian angular resolution," Phys. Rev. Lett. 110(13), 138105 (2013).

[21] Diemoz, P. C., Hagen, C. K., Endrizzi, M. and Olivo, A., "Sensitivity of laboratory based implementations of edge illumination x-ray phase-contrast imaging," Appl. Phys. Lett. 103(24), 244104 (2013).

[22] Munro, P. R. T., Ignatyev, K., Speller, R. D. and Olivo, A., "Phase and absorption retrieval using incoherent xray sources," Proc. Natl. Acad. Sci. USA 109(35), 13922-7 (2012).

[23] Munro, P. R. T., Hagen, C. K., Szafraniec, M. B. and Olivo, A., “A simplified approach to quantitative coded aperture x-ray phase imaging," Opt. Exp. 21(9), 11187-201 (2013).

[24] Ruan, M. Z. C., Dawson, B., Jiang, M.-M., Gannon, F., Heggeness, M. and Lee, H. L., "Quantitative imaging of murine osteoarthritic cartilage by phase-contrast micro-computed tomography," Arthritis Rheum. 65(2), 388-96 (2013).

[25] Gennaro, G., Baldelli, P., Taibi, A., Di Maggio, C. and Gambaccini, M., "Patient dose in full-field digital mammography: an Italian survey," Eur. Radiol. 14(4), 645-52 (2004).

[26] Thüring, T., Hämmerle, S., Weiss, S., Nüesch, J., Meiser, J., Mohr, J., David, C. and Stampanoni, M., "Compact hard x-ray grating interferometry for table top phase contrast micro CT," Proc. SPIE 8668, 866813 (2013).

[27] Revol, V., Kottler, C., Kauffman, R., Straumann, U. and Urban, C. "Noise analysis of grating-based x-ray differential phase contrast imaging," Rev. Sci. Instrum. 81(7), 073709 (2010).

[28] Endrizzi, M., Vittoria, F. A., Diemoz, P. C., Lorenzo, R., Speller, R. D., Wagner, U., Rau, C., Robinson, I. K. and Olivo A., "Quantitative phase-contrast microscopy at high X-ray energy with a laboratory set-up," under review. 